\section{CVIA}

ORIGINAL ARTICLE

pISSN 2508-707X / eISSN 2508-7088 https://doi.org/10.22468/cvia.2018.00108 CVIA 2018;2(4):187-193

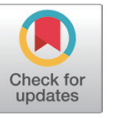

\title{
Global Left Ventricular Area Strain Using Standard Two-Dimensional Cine Magnetic Resonance Imaging with Inter-Slice Interpolation
}

\author{
Masateru Kawakubo', Hideo Arai², Michinobu Nagao³, \\ Yuzo Yamasaki ${ }^{4}$, Kenichi Sanui ${ }^{2}$, Hiroshi Nishimura², \\ Toshiaki Kadokami \\ 'Department of Health Sciences, Faculty of Medical Sciences, Kyushu University, Fukuoka, Japan \\ ${ }^{2}$ Fukuokaken Saiseikai Futsukaichi Hospital, Fukuoka, Japan \\ ${ }^{3}$ Department of Diagnostic Imaging \& Nuclear Medicine, Tokyo Women's Medical University, \\ Tokyo, Japan \\ ${ }^{4}$ Department of Clinical Radiology, Graduate School of Medical Sciences, Kyushu University, \\ Fukuoka, Japan
}

\begin{abstract}
Objective: We proposed the use of standard two-dimensional (2D) cine MR images with inter-slice interpolation to evaluate three-dimensional (3D) left ventricular (LV) global area strain (GAS), and investigated its clinical applicability by comparing to 2D strains.

Materials and Methods: Twenty-one consecutive patients with ischemic cardiomyopathy

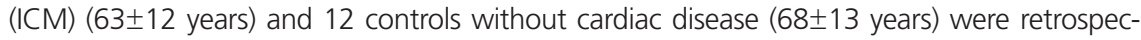
tively enrolled. Receiver operating characteristic analysis was performed to determine the optimal cutoff of circumferential strain (CS), radial strain (RS), longitudinal strain (LS), and GAS for detection of patients with LV ejection fraction (LVEF) $\leq 45 \%$. Comparison of CS, RS, LS, and GAS between controls and ICM patients was analyzed by the Wilcoxon rank-sum test.

Results: The optimal cutoff of GAS has the highest diagnostic performance for detection of patients with LVEF $\leq 45 \%$ [area under the curve (AUC), 0.97; sensitivity, 88\%; specificity, $100 \%$ ] compared to the other 2D strains (CS vs. RS vs. LS; AUC, 0.85 vs. 0.85 vs. 0.73 ; sensitivity, $75 \%$ vs. $100 \%$ vs. $50 \%$; specificity, $85 \%$ vs. $77 \%$ vs. $85 \%$ ). LS and GAS were significantly greater for controls than ICM patients (LS: $-18.6 \pm 3.2 \%$ vs. $-10.1 \pm 3.2 \%, p<0.01, G A S:-41.3 \pm$ $5.3 \%$ vs. $-26.0 \pm 8.2 \%, p<0.01)$.
\end{abstract}

Conclusion: GAS derived from inter-slice interpolation of 2D cine MRI is a quantitative index of 3D myocardial motion, and this technique can be applied for clinical MR examinations.

Key words Myocardial contraction - Ischemia - Cardiomyopathy · Cine Magnetic Resonance Imaging · Left ventricle.

Received: April 20, 2018

Revised: June 20, 2018

Accepted: August 7, 2018

Corresponding author

Department of Health Sciences,

Faculty of Medical Sciences,

Kyushu University, 3-1-1 Maidashi,

Higashi-ku, Fukuoka 812-8582, Japan

Tel: 81-92-642-6728

Fax: 81-92-642-6728

E-mail: k-mstr@hs.med.kyushu-u.ac.jp

\section{INTRODUCTION}

Feature tracking magnetic resonance imaging (FT-MRI) allows tracking of tissue voxel motion using only the standard twodimensional (2D) cine MRI and can be used to evaluate wall mechanics and strains without acquisition of additional sequences [1]. FT-MRI has been validated against myocardial tagging with

(a) This is an Open Access article distributed under the terms of the Creative Commons Attribution Non-Commercial License (https://creativecommons.org/licenses/bync/4.0) which permits unrestricted non-commercial use, distribution, and reproduction in any medium, provided the original work is properly cited. harmonic phase imaging [2], and the usefulness of strain value as a clinical indicator [3-7]. However, FT-MRI has the intrinsic limitation of being 2D. A fixed 2D slice plane loses visual information from the original myocardial features during frame-byframe tracking analysis [8]. This problem is encountered in 2D feature-tracking analysis using both the $2 \mathrm{D}$ short-axis and longaxis views. Three-dimensional (3D) FT-MRI has the theoretical advantage of overcoming through-plane motion. Specifically, the left ventricular (LV) surface area decreases in size because of circumferential, radial, and longitudinal shortening. Area strain re- 
flects these changes in endocardial surface area and quantifies them as the percentage change in area relative to the original dimensions at the end-diastole phase [9]. Global area strain (GAS) measured using echocardiography, which quantifies the percentage of change in endocardial surface area, can be a sensitive marker of myocardial damage [10]. Area strain analysis using 3D FT-MRI potentially provides more detailed information regarding myocardial deformation and damage by taking advantage of a wide field of view. However, during clinical MR examination, there are some limitations for acquiring 3D cinematic data due to the long acquisition time and increased patient burden due to repeated breath holds. Previously, several inter-slice interpolation techniques have been reported for medical imaging analysis [11-13]. We hypothesized that standard 2D cine MR data can be interpolated as volumetric image data acquired by these techniques. In this study, we proposed LV GAS analysis using only standard 2D cine MR images with inter-slice interpolation and investigated the clinical applicability.

\section{MATERIALS AND METHODS}

\section{Patient population}

Twenty-one consecutive patients with ischemic cardiomyopathy (ICM) who underwent MR examination including shortaxis and long-axis cine MRI (age: $63 \pm 12$ years; 20 men and 1 woman) were retrospectively enrolled. The detailed characteristics of the patients with ICM are shown in Table 1. The diagnoses were performed by two experienced cardiologists in consensus using all available information [data from medical history, electrocardiogram (ECG), laboratory tests, coronary angiography, cardiac computed tomography] as well as cardiovascular magnetic resonance results (cine and late gadolinium enhancement). All patients were clinically stable (New York Heart Association functional class $\leq \mathrm{III}$ ) and presented with sinus rhythms during the MR examination. In addition, 12 patients who were diagnosed with normal cardiac function and no prior cardiac disease by routine MR examination (age, $68 \pm 13$ years; 4 men and 8 women) were retrospectively enrolled as controls. Detailed characteristics of the control patients are also shown in Table 1. The study protocol was reviewed and approved by the Institutional Review Board (No. 207); the study was conducted in accordance with the Declaration of Helsinki. Written informed consent was obtained from all patients.

\section{Cine MRI parameters}

All MR examinations were performed using a 3.0-T clinical scanner (Ingenia 3.0 T, Philips Healthcare, Best, the Netherlands), equipped with a cardiac phased array 32-channel receiver coil. Cine MRI with steady-state free precession sequence was obtained using multi-breath holding (lasting approximately 10-
Table 1. Characteristics in study population

\begin{tabular}{lccc}
\hline & $\begin{array}{c}\text { Controls } \\
(\mathrm{n}=12)\end{array}$ & $\begin{array}{c}\text { ICM } \\
(\mathrm{n}=21)\end{array}$ & p value \\
\hline Clinical characteristics & & & \\
Age $(\mathrm{y})$ & $68 \pm 13$ & $63 \pm 12$ & $\mathrm{NS}$ \\
Men/women & $4 / 8$ & $20 / 1$ & \\
Hear rate (beats/min) & $58 \pm 11$ & $65 \pm 7$ & 0.008 \\
Body surface area $\left(\mathrm{m}^{2}\right)$ & $1.6 \pm 0.2$ & $1.9 \pm 0.2$ & $\mathrm{NS}$ \\
LV function with MRI & & & \\
LVEDVI $\left(\mathrm{mL} / \mathrm{m}^{2}\right)$ & $70 \pm 12$ & $79 \pm 22$ & $\mathrm{NS}$ \\
LVESVI $\left(\mathrm{mL} / \mathrm{m}^{2}\right)$ & $26 \pm 7$ & $47 \pm 19$ & 0.001 \\
LVEF $(\%)$ & $63 \pm 7$ & $42 \pm 10$ & $<0.001$ \\
\hline
\end{tabular}

Data are expressed as mean \pm standard deviation or as number. ICM: ischemic cardiomyopathy, LV: left ventricular, LVEDVI: LV end-diastolic volume index, LVESVI: LV end-systolic volume index, LVEF: LV ejection fraction

20 s), retrospective ECG gating in the 4-chamber view, and contiguous short-axis views covering the whole LV with 20 phases per cardiac cycle. To prevent image misalignment due to breathhold defects, sufficient explanation and training for breathing were provided to all patients before the MR examination. Typical cine imaging parameters were as follows: repetition time $=3.2$ $\mathrm{ms}$, echo time $=1.6 \mathrm{~ms}$, flip angle $=50^{\circ}$, slice thickness $=8.0 \mathrm{~mm}$, field of view $=350 \times 350 \mathrm{~mm}^{2}$, acquisition matrix $=176 \times 176$ pixels, and reconstruction matrix $=352 \times 352$ pixels.

\section{Strain analysis using $2 \mathrm{D}$ cine MRI}

The 2D strain analyses were performed following manual correction on short-axis and 4-chamber MR images of the middle $\mathrm{LV}$ using the original off-line feature tracking tool. This original feature tracking algorithm was previously validated in a clinical study [14]. First, endocardial borders were manually defined as some arbitrary points at the end of diastole. Next, the endocardium points were tracked for one cardiac cycle with a local template-matching technique based on normalized correlation coefficient values. Finally, the endocardial strain values for one cardiac cycle were automatically calculated from the coordinates of each point. From the strain analysis with short-axis images, minimum circumferential strain (CS) and minimum radial strain (RS) of LV were obtained. Similarly, minimum longitudinal strain (LS) of LV was obtained from 4-chamber images.

\section{Global area strain analysis using 2D cine MRI with inter-slice interpolation}

Our proposed area strain analysis using standard 2D MRI with inter-slice interpolation consisted of 3 steps (Fig. 1). First, the $2 \mathrm{D}$ cine $\mathrm{MR}$ images were inter-slice interpolated to the $3 \mathrm{D}$ cine MR images with $1.0 \mathrm{~mm}$ slice thickness using a path-based moving gradient technique [12]. This interpolation technique provides an arbitrary number of interpolated images from the 
moving gradients of 2 images for up-sampling of low-frame videos and interpolating viewpoints between images captured at different camera locations. In this study, two short-axis images in adjacent slice positions were interpolated by 7 images. This interpolation was performed in all slice positions through an entire cardiac phase. Second, on all slices of 2D MR short-axis images of the end of diastole, the LV endocardium border was defined as any number of points. This marking of the endocar- dial borders was performed only on the stacked MR images and not on the interpolated MR images. Then, the template image was set as the circumscribed rectangle of the defined points. The template size was manually set in each case. A cross-correlation analysis was performed between the template image and the interpolated images. Interpolated MR images with slice positions above and below that of the template were selected for matching based on the slice position of the selected template image with

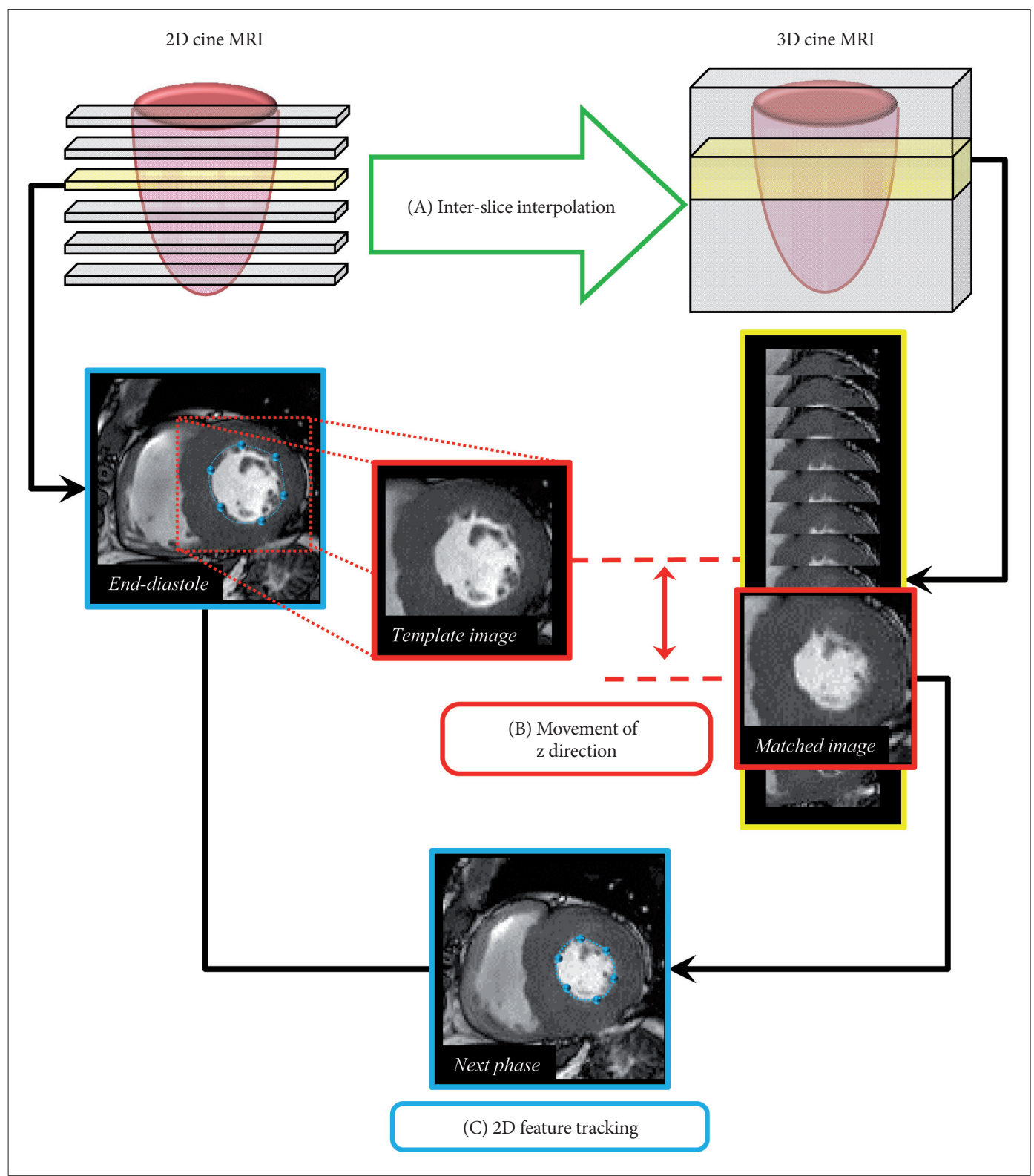

Fig. 1. First, the $2 D$ cine $M R$ images were inter-slice interpolated to the $3 D$ cine $M R$ images (green frame arrow) (A). Second, on the 2D MR short-axis images at end-diastole, the LV endocardium border was defined as any number of points (upper blue frame image). Then, the template image was set as the circumscribed rectangle of the defined points (left red frame image). Cross-correlation analysis was performed between the template image and the interpolated images (yellow frame images), and the best matched image was defined (right red frame image). Longitudinal myocardial movement was calculated using the differences in slice position between the template image and the best-matched image (B). Finally, points on the LV endocardium border were tracked on the matched image with local template-matching technique (lower blue frame image) (C). These points were automatically tracked for one cardiac cycle. 2D: two-dimensional, 3D: three-dimensional, LV: left ventricular. 
the marked endocardium. The number of interpolated images for matching was manually set in each case. The best-matched image was defined as the one with the highest normal crosscorrelation value relative to the template image. Longitudinal myocardial movement was calculated according to the differences in slice position between the template image and the bestmatched image. Finally, points on the LV endocardium border were tracked on the matched image with local template-matching technique. The points of the LV endocardium were automatically tracked for a cardiac cycle. If the points were incorrectly defined by automatic tracking, the positions of the points were corrected manually. This longitudinal and temporal tracking of points was performed in all 2D short-axis images. In this way, an arbitrary number of 3D coordinates on the myocardial surface was obtained through a cardiac cycle. The endocardial areas were calculated for one cardiac cycle by summing the product of the LV perimeter with the slice thickness across all 2D short- axis cine MR images, analogous to Simpson's method for calculating the global LV volume. The area strain was calculated as the percentage change in area relative to the original area at end-diastole. GAS was defined as the minimum value of area strain for one cardiac cycle.

\section{Intra- and inter-observer reproducibility}

All strain measurements (CS, RS, LS, and GAS) were tested for intra-observer reproducibility by having one observer perform all GAS analyses on 10 randomly selected patients and then blindly repeating the analysis on a separate occasion. Interobserver reproducibility was evaluated by a second observer, blinded to clinical and experimental data, performing strain measurements on the same 10 patients. The intra- and inter-observer reproducibility values of strain measurements were evaluated as the absolute value of the difference between each pair of measurements (by the same observer or different observers)
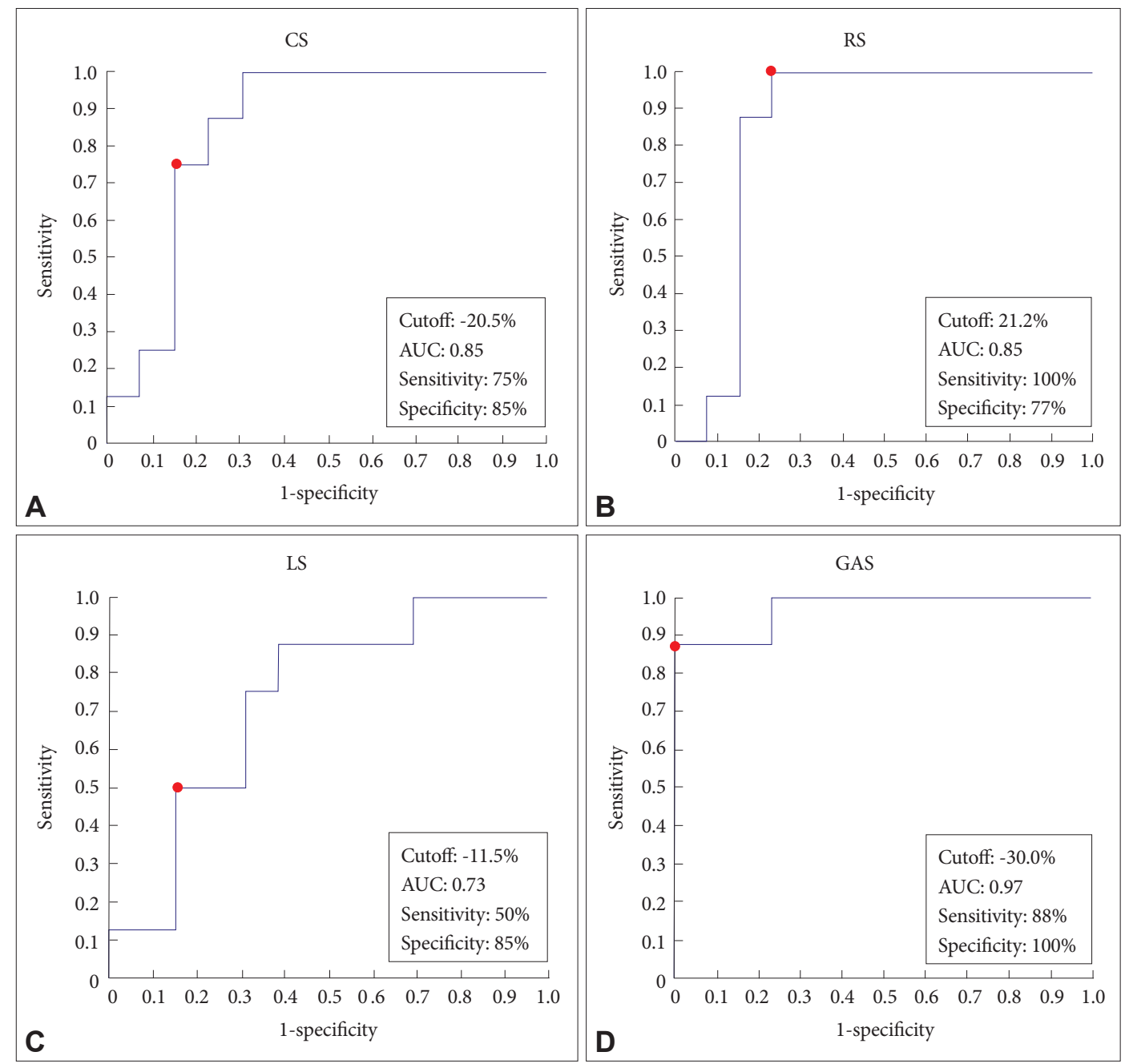

Fig. 2. ROC curves for the CS (A), RS (B), LS (C), and (D) GAS for identifying patients with LVEF $\leq 45 \%$. ROC analysis of revealed that the optimal cutoff of the GAS (-30.0\%) identified patients with LVEF $\leq 45 \%$, with AUC of the widest 0.97 in all strains, $88 \%$ sensitivity, and $100 \%$ specificity. ROC: receiver operating characteristic, CS: circumferential strain, RS: radial strain, LS: Iongitudinal strain, GAS: global area strain, AUC: area under the curve, LVEF: left ventricular ejection fraction. 
divided by the mean of the measurement pair (expressed as a percentage).

\section{Statistical analysis}

Continuous data were expressed as the mean \pm standard deviation. Receiver operating characteristic (ROC) analysis was performed to determine the optimal cutoff of strain for detection of patients with $\mathrm{LV}$ ejection fraction (LVEF) $\leq 45 \%$. The CS, RS, LS, and GAS were compared using the Wilcoxon rank-sum test between the control group $(n=12)$ and ICM group $(n=21)$. In the ICM group, the Pearson correlation coefficients (r) between LVEF and the CS, RS, LS, and GAS were calculated. The Pearson correlation coefficients (r) of GAS with respect to CS, RS, and LS were calculated. All statistical analyses were conducted using SAS software for Windows (version 13, SAS Inc., Cary, NC, USA). Statistical significance was set at $\mathrm{p}<0.05$.

\section{RESULTS}

ROC analysis revealed the optimal cutoff of GAS for identifying patients with LVEF $\leq 45 \%$ [GAS $=-30.0 \%$ : area under the curve (AUC), 0.97; sensitivity, 88\%; specificity, 100\%]. Details of the results of ROC analysis are shown in Fig. 2. LS and GAS were significantly greater for controls than ICM patients (LS: $-18.6 \pm 3.2 \%$ vs. $-10.1 \pm 3.2 \%, \mathrm{p}<0.01$, GAS: $-41.3 \pm 5.3 \%$ vs. $-26.0 \pm 8.2 \%, \mathrm{p}<0.01$ ) (Fig. 3). There were no significant differences in the CS and RS between the two groups (CS: -19.8 \pm $5.1 \%$ vs. $-17.2 \pm 7.4 \%$, RS: $23.2 \pm 5.5 \%$ vs. $19.0 \pm 7.2 \%$ ). The CS, RS, LS, and GAS were significantly correlated with LVEF (CS: $\mathrm{r}=-0.59, \mathrm{p}<0.01$, RS: $\mathrm{r}=0.65, \mathrm{p}<0.01$, LS: $\mathrm{r}=-0.50, \mathrm{p}<0.05$, and GAS: $r=-0.90, p<0.01$ ) in the ICM group (Fig. 4). The correlation of GAS to LVEF was significantly stronger than those of CS, RS, and LS. The GAS was significantly correlated with CS, RS, and LS (CS: $r=0.52, p<0.05$, RS: $r=-0.58, p<0.01$, and LS: $r=$ $0.51, \mathrm{p}<0.05)$. Excellent intra- and inter-observer reproducibility was seen for all strain measurements (Table 2 ).

\section{DISCUSSION}

In the present study, ROC analysis showed the highest diagnostic performance of GAS for detecting LVEF $\leq 45 \%$ for all strains. With the comparison between 2D strains (CS, RS, and LS) and 3D GAS in the controls and ICM patients, significant degradation of LS and GAS in ICM patients was observed. In ICM patients, GAS had the strongest statistical correlation with LVEF for all strains. Moreover, GAS was significantly correlat-
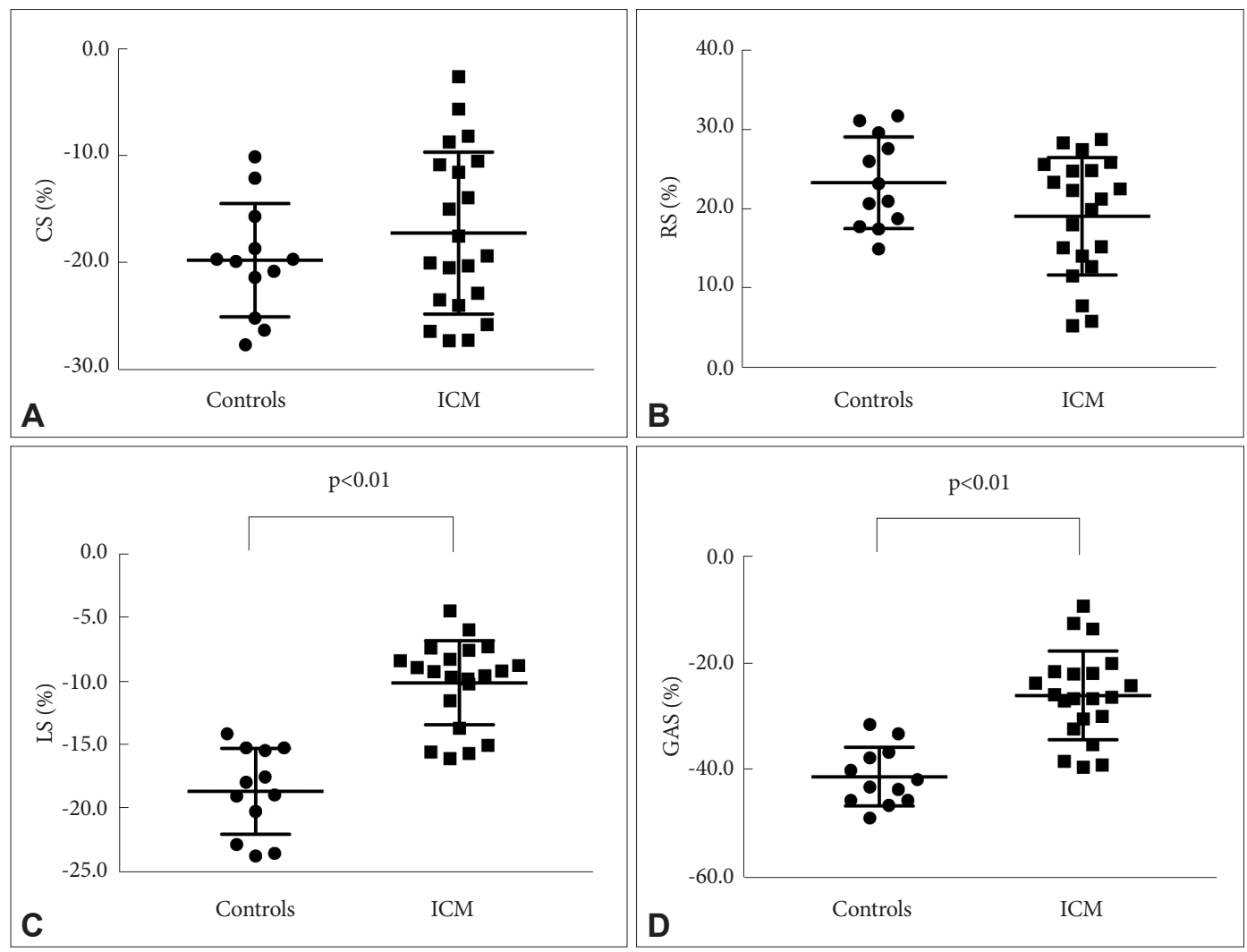

Fig. 3. Scatter plot of the strains between the controls and patients with ICM. There were significant differences in CS (A), RS (B), LS (C), and GAS (D) between the two groups (CS: $-19.8 \pm 5.1 \%$ vs. $-17.2 \pm 7.4 \%$, RS: $23.2 \pm 5.5 \%$ vs. $19.0 \pm 7.2 \%$, LS: $-18.6 \pm 3.2 \%$ vs. $-10.1 \pm 3.2 \%$, p<0.01, GAS: $-41.3 \pm 5.3 \%$ vs. $-26.0 \pm 8.2 \%$, p<0.01). ICM: ischemic cardiomyopathy, CS: circumferential strain, RS: radial strain, LS: longitudinal strain, GAS: global area strain. 

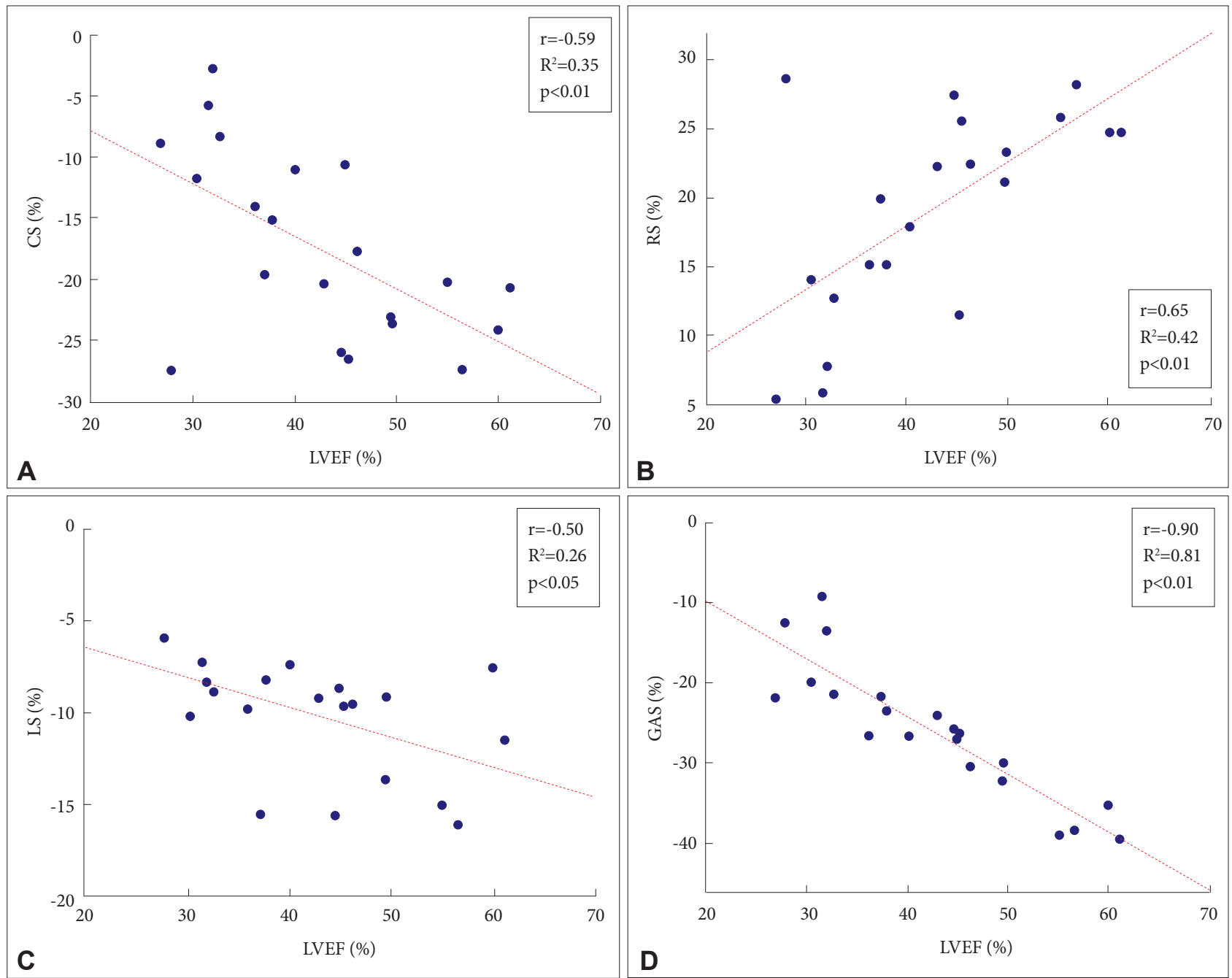

Fig. 4. CS (A), RS (B), LS (C), and GAS (D) were significantly correlated with LVEF (CS: $r=-0.59$, $p<0.01, R S$ : $r=0.65$, $p<0.01$, LS: $r=-0.50$, $p<0.05$, and GAS: $r=-0.90, p<0.01)$ in patients with ICM. The correlation of GAS with LVEF was stronger than those of CS, RS, and LS. CS: circumferential strain, RS: radial strain, LS: longitudinal strain, GAS: global area strain, ICM, ischemic cardiomyopathy, LVEF: left ventricular ejection fraction.

Table 2. Intra- and inter-observer reproducibility

\begin{tabular}{lcccc}
\hline & GAS & CS & RS & LS \\
\hline Intra-observer reproducibility (\%) & $1.5 \pm 1.1$ & $1.4 \pm 0.9$ & $1.9 \pm 1.3$ & $1.6 \pm 0.9$ \\
Inter-observer reproducibility (\%) & $1.6 \pm 1.2$ & $1.2 \pm 1.0$ & $1.9 \pm 1.4$ & $1.8 \pm 1.2$ \\
\hline
\end{tabular}

GAS: global area strain, CS: circumferential strain, RS: radial strain, LS: longitudinal strain

ed with CS, RS, and LS. These results indicate that GAS is correlated with 2D strains and reflects 3D myocardial motion better than 2D strain. Technically, our proposed analysis for global myocardial strains can be performed using only the standard 2D cine short-axis MRI without additional data acquisition. Therefore, measurement of area strain using MRI is easily applicable during routine MR examination. Recently, 3D cine MR data acquisition with a compressed sensing technique was reported [15-19]. Our analytic algorithm can be applied to 3D cine MR data. Our area strain analysis is expected to improve with general application of high-speed MRI with compressed sensing.
In the intra- and inter-observer analyses, area strain analysis exhibited excellent reproducibility. Moreover, Kleijn et al. [9] have reported that normal reference values of LV global and regional area strain in healthy populations $(\mathrm{n}=56)$ are approximately $-40 \%$. Our results showed that area strains in controls were also approximately $-40 \%$. This concurrence of results supports the accuracy of our proposed area strain analysis. Therefore, area strain analysis is a robust method with reproducibility equivalent to that of $2 \mathrm{D}$ strain analysis with FT-MRI. 


\section{Limitations}

There are some limitations in the present study. The regional area strain analysis could not be validated in the patient group. In future research, the detection accuracy of the myocardial scar area should be verified for patients with decreased wall motion. The other limitation is the small size of the study population. This was a preliminary study of the proposed technique to evaluate the GAS applied to patients with ICM. However, the measurements of area strain in the control group were in agreement with those from previous studies. Our technique can accurately evaluate GAS. Apart from these limitations, this study lacks validation of the application of the path-based interpolation method to clinical MRI. This was the reason that all patients in this study could hold their breath with high reproducibility. In actuality, imaging conditions, such as the acceptance and reproducibility of patient breath holds and slice widths, affect the accuracy of image interpolation. Therefore, explanations and training before MR examinations for breath holding are recommended for inter-slice interpolation, as we performed in this study.

In the present study, we proposed a technique for evaluation of LV GAS with standard 2D cine MRI with inter-slice interpolation. The area strain analysis with MRI is useful and clinically applicable.

\section{Conflicts of Interest}

The authors declare that they have no conflict of interest.

\section{Acknowledgments}

This work was supported by JSPS KAKENHI Grant Number JP16K19860.

\section{REFERENCES}

1. Hor KN, Baumann R, Pedrizzetti G, Tonti G, Gottliebson WM, Taylor $\mathrm{M}$, et al. Magnetic resonance derived myocardial strain assessment using feature tracking. J Vis Exp 2011;48:e2356.

2. Hor KN, Gottliebson WM, Carson C, Wash E, Cnota J, Fleck R, et al. Comparison of magnetic resonance feature tracking for strain calculation with harmonic phase imaging analysis. JACC Cardiovasc Imaging 2010;3:144-151.

3. Choi EY, Rosen BD, Fernandes VR, Yan RT, Yoneyama K, Donekal S, et al. Prognostic value of myocardial circumferential strain for incident heart failure and cardiovascular events in asymptomatic individuals: the Multi-Ethnic Study of Atherosclerosis. Eur Heart J 2013;34:2354-2361.

4. Gavara J, Rodriguez-Palomares JF, Valente F, Monmeneu JV, Lopez-Lereu MP, Bonanad C, et al. Prognostic value of strain by tissue tracking cardiac magnetic resonance after ST-segment elevation myocardial infarction. JACC Cardiovasc Imaging 2017 Dec 8 [Epub]. https://doi.org/10.1016/ j.jcmg.2017.09.017.

5. Yang LT, Yamashita E, Nagata Y, Kado Y, Oshima S, Otsuji Y, et al. Prog- nostic value of biventricular mechanical parameters assessed using cardiac magnetic resonance feature-tracking analysis to predict future cardiac events. J Magn Reson Imaging 2017;45:1034-1045.

6. Hinojar R, Fernández-Golfín C, González-Gómez A, Rincón LM, PlazaMartin M, Casas E, et al. Prognostic implications of global myocardial mechanics in hypertrophic cardiomyopathy by cardiovascular magnetic resonance feature tracking. Relations to left ventricular hypertrophy and fibrosis. Int J Cardiol 2017;249:467-472.

7. Buss SJ, Breuninger K, Lehrke S, Voss A, Galuschky C, Lossnitzer D, et al. Assessment of myocardial deformation with cardiac magnetic resonance strain imaging improves risk stratification in patients with dilated cardiomyopathy. Eur Heart J Cardiovasc Imaging 2015;16:307-315.

8. Wu VC, Takeuchi M, Otani K, Haruki N, Yoshitani H, Tamura M, et al. Effect of through-plane and twisting motion on left ventricular strain calculation: direct comparison between two-dimensional and three-dimensional speckle-tracking echocardiography. J Am Soc Echocardiogr 2013; 26:1274-1281.e4.

9. Kleijn SA, Aly MF, Terwee CB, van Rossum AC, Kamp O. Three-dimensional speckle tracking echocardiography for automatic assessment of global and regional left ventricular function based on area strain. J Am Soc Echocardiogr 2011;24:314-321.

10. Kowalik E, Kowalski M, Klisiewicz A, Hoffman P. Global area strain is a sensitive marker of subendocardial damage in adults after optimal repair of aortic coarctation: three-dimensional speckle-tracking echocardiography data. Heart Vessels. 2016;31:1790-1797.

11. Mahmoudzadeh AP, Kashou NH. Interpolation-based super-resolution reconstruction: effects of slice thickness. J Med Imaging (Bellingham) 2014;1:034007.

12. Mahajan D, Huang FC, Matusik W, Ramamoorthi R, Belhumeur P. Moving gradients: a path-based method for plausible image interpolation. ACM Transactions on Graphics 2009;28:42.

13. Albu AB, Beugeling T, Laurendeau D. A morphology-based approach for interslice interpolation of anatomical slices from volumetric images. IEEE Trans Biomed Eng 2008;55:2022-2038.

14. Kawakubo M, Nagao M, Kumazawa S, Yamasaki Y, Chishaki AS, Nakamura Y, et al. Evaluation of ventricular dysfunction using semi-automatic longitudinal strain analysis of four-chamber cine MR imaging. Int J Cardiovasc Imaging 2016;32:283-289.

15. Wech T, Pickl W, Tran-Gia J, Ritter C, Beer M, Hahn D, et al. Wholeheart cine MRI in a single breath-hold--a compressed sensing accelerated 3D acquisition technique for assessment of cardiac function. Rofo 2014;186:37-41.

16. Vincenti G, Monney P, Chaptinel J, Rutz T, Coppo S, Zenge MO, et al. Compressed sensing single-breath-hold CMR for fast quantification of LV function, volumes, and mass. JACC Cardiovasc Imaging 2014;7:882892.

17. Usman M, Atkinson D, Odille F, Kolbitsch C, Vaillant G, Schaeffter T, et al. Motion corrected compressed sensing for free-breathing dynamic cardiac MRI. Magn Reson Med 2013;70:504-516.

18. Goebel J, Nensa F, Schemuth HP, Maderwald S, Gratz M, Quick HH, et al. Compressed sensing cine imaging with high spatial or high temporal resolution for analysis of left ventricular function. J Magn Reson Imaging 2016;44:366-374.

19. Kido T, Kido T, Nakamura M, Watanabe K, Schmidt M, Forman C, et al. Compressed sensing real-time cine cardiovascular magnetic resonance: accurate assessment of left ventricular function in a single-breath-hold. J Cardiovasc Magn Reson 2016;18:50. 